\title{
A case study to the Evaluation of Global Environmental Quality, concerning thermal, acoustic and atmospheric conditions of urban areas.
}

\author{
V. Franzitta ${ }^{1, a}$, A. Milone $e^{1, b}, D$. Milone $^{1, c}, M$. Trapanese $^{1 d,}$, A. Viola ${ }^{1, e}$ \\ ${ }^{1}$ DEIM -Dipartimento Di Energia, Ingegneria Dell'informazione, E Modelli Matematici, University of \\ Palermo, Palermo 90128, Italy \\ afranzitta@dream.unipa.it , bangelo.milone@unipa.it 'daniele.milone@unipa.it, \\ dmarco.trapanese@unipa.it, ${ }^{\mathrm{e}}$ alessia.viola@unipa.it
}

Keywords: air pollution; noise quality; emissions;

\begin{abstract}
In this paper we propose an operative procedure aimed at assessing and check the global environmental quality in the urban areas. This methodology is set up by using an ergonomic approach that analyses the interaction man-environment in terms of cause-effect and uses information and data from various sources contributing to the formation of discomfort degrees.[1-2] The representation technique by normo-grams is used for showing some applications executed along the urban territory of Palermo and allows to evaluate very simply all the parameters involved in human comfort. This paper is divided in 5 sections, after the introduction in section 2 a review of the principal simple and complex indexes is presented, in section 3 the methodology used to assess the air quality is presented and in section 4 some experimental results on some areas of Palermo are presented, finally in section 5 some conclusions are drawn.[3-4]
\end{abstract}

\section{Introduction}

In order to satisfy human needs in both outdoor and indoor spaces, environmental evaluation and design play a key role in urban planning. This paper proposes a global approach to the evaluation of the outdoor environmental quality. In order to have a simple determination of the structure and output of the methodology only the thermal, acoustic and atmospheric components of urban climate using hourly data from a measurement ground network are used to characterize the urban environment. More particularly, we use data of two measurement stations describing different microclimatic behavior: one is located in an urban area (Castelnuovo Square) and the other in a rural area(Boccadifalco Airport). The mathematical tools used to analyze these data have been used by the authors in other fields of science [5-14]. In order to get a deeper evaluation, the data obtained are used to compute the following indirect composite descriptors. These descriptors describe respectively the thermal, acoustic and air pollution levels. This approach allows to evaluate the compatibility man-environment in terms of a synthesis of human sensation, stress levels, health effects, comfort assessment corresponding to selected ranges of values of the aforesaid composite indexes. Results are showed as maps of the comfort hours or days during a monthly sequence.

Atmospheric and thermal effective complex. The theoretical clean air is composed mainly from nitrogen, oxygen and from small concentrations of other agents. It is however at a various level contaminated from the presence of other gases and other liquid and solid particles. When the concentration of such agents is able to modify the physical chemical property of the air with a potential risk for the exposed population, we can speak of atmospheric pollution.

The EPA's Pollutant Standard Index (PSI) (Wayne 1978) is calculated according to the following equation [1] as a function of four guide pollutants sulphur dioxide, nitrogen oxide, carbon monoxide and particulate: 


$$
P S I=\left[\sum_{1}^{4} m P_{i}^{k}\right]^{k-1}
$$

where:

$\mathrm{P}_{\mathrm{i}}=$ Ratio between atmospheric levels of pollutant "i" and its air quality standard,

$\mathrm{m}=$ weighing factor

$\mathrm{K}=2$

The Air Quality Stress Index AQSI is defined according to Baümuller et al. [2], which integrates information from four prevailing non-carcinogenic pollutants (atmospheric contents of carbon monoxide $\mathrm{CO}$, nitrogen dioxide $\mathrm{NO}$, dust, $\mathrm{SO}$ ) weighting the ratio to the corresponding standards:

$$
A Q S I_{24}=\sum_{i=1}^{4}\left(\frac{X_{d, i}}{S_{d, i}}\right)
$$

where AQSI 24 is the air quality stress related to the a whole day, $\mathrm{X}_{\mathrm{d}, \mathrm{I}}$ and $\mathrm{S}_{\mathrm{d}, \mathrm{I}}$ are respectively the average daily concentration of the its pollutant and the corresponding threshold value for $24 \mathrm{~h}$ of the its pollutant. Man's activities have likely a significant influence on climate. This influence, local or global, is difficult to predict because the exchanges of energy, mass, and momentum are very complex. Urbanization has been increasingly responsible for dramatic climatic changes. The city's compact mass of buildings and pavement exhibits a complex geometry of streets canyons and a large spatial heterogeneity, and constitutes a profound alteration of the natural landscape, resulting in a large number of micro-climates. These micro-climates may be revealed by the existence of the so-called urban heat islands where changes in the temperature of the atmosphere may reach several degrees compared to that of the neighbour rural area.

Urban planners, decision-makers and city leaders, as well as responsible officials of the environment or health care, need a comprehensive overview of the urban climate in order to understand the results of their urban policy, to assess the effects of air pollution, as well as to foresee the effects of new regulations.

The climatic quality analysis of a territory can be performed by using the simple indices directly measured and the composites indices that allow to estimate the conditions of thermal and hygrometric comfort in the open spaces.

Acoustical complex. The main sources of urban acoustic pollution are classified in fixed sources and moving sources. Fixed sources are: technical systems of buildings and the other installations joined to the buildings also in transitory way whose use produces sonorous emissions; street, railway, airport, marine, industrial, handicraft, commercial and agricultural infrastructures; parking; warehouses for persons and freights transport means; areas used to recreational and sport activity.

Mobile sources are the road, railway, airport and marine traffic. The first constitutes anyway the more important source both for the high noise levels and the space and time wide diffusion, affecting the whole urban area (territory and people) with negative environmental effects.

A direct simple index for the acoustical quality analysis is the Equivalent Sound Level at a basis instantaneous or hourly.

The A-weighted sound level that is "equivalent" to an actual time varying sound level, in the sense that it has the same total energy for the duration of the sound.

The computed average values of continuous equivalent level for the reference day Ld and night Ln are calculated using the following formulas:

$L_{d}=10 * \log \left[\frac{1}{16} * \sum_{i=1}^{16} 10^{\left(0.1 * L e q_{h i}\right)}\right]$ 
$L_{n}=10 * \log \left[\frac{1}{b} * \sum_{i=1}^{2} 10^{\left(0,1 * L E q_{h}, i\right)}\right] \quad[d B A]$

where $\mathrm{L}_{\mathrm{eqh}}, \mathrm{i}$ is the average hourly continuous equivalent level.

The Italian rules impose different equivalent levels for different zones of the urban territory as showed in the following Table 1.

Table 1. Acoustic zoning of urban territory limits

\begin{tabular}{|l|l|l|}
\hline \multicolumn{1}{|c|}{ Urban area classification } & \multicolumn{1}{c|}{$\begin{array}{c}\text { Maximum dayly level } \\
\text { Ld }\end{array}$} & $\begin{array}{c}\text { Maximum nightly level } \\
\text { Ln }\end{array}$ \\
\hline Whole national territory & 70 & 60 \\
\hline Zone A (historic centre art.2 of D.M. 02/04/68, n.1444) & 65 & 55 \\
\hline $\begin{array}{l}\text { Zone B (Residential Zone,art.2 of D.M. } \\
\text { 02/04/68,n.1444) }\end{array}$ & 60 & 50 \\
\hline Industrial Area & 70 & 70 \\
\hline
\end{tabular}

Methodology. The Metropolitan area of Palermo is characterized by quite a complex geo-topography: Palermo is located on a flat area called "Conca d'oro" close to the Tyrrhenian sea and delimitated at west and south by a series of mountains with an altitude ranging between 700 and 1000 meters over the sea level.

In order to assess the environment quality several monitoring stations in partnership with Municipal Company of Environmental Hygiene (AMIA network) have been placed in environmental meaningful sites. The objectives of this network are :

- comparing the collected atmospheric data with the standard imposed by the enforced rules;

- informing the citizens about the reached pollution levels;

- providing wide and spread information useful for finding the causes and characterizing the source of pollution, their dynamic and the critical periods;

- supplying an effective planning and environmental management tool of the territory.

Experimental Results. An application of the above mentioned procedure has been executed on a monthly base of the year 2000. In this paper, only the data of two measurement stations have been used and the normo-grams for the environmental and the acoustic comfort have been presented.

A criterion for the thermo-atmospheric classification of the human comfort is proposed that expresses in a graph alone the information provided by the indices THI and AQSI. In the following figure is showed the aforesaid nomo-gram in correspondence of the data collected in two monitoring stations in the time interval 24 January - 7 February.

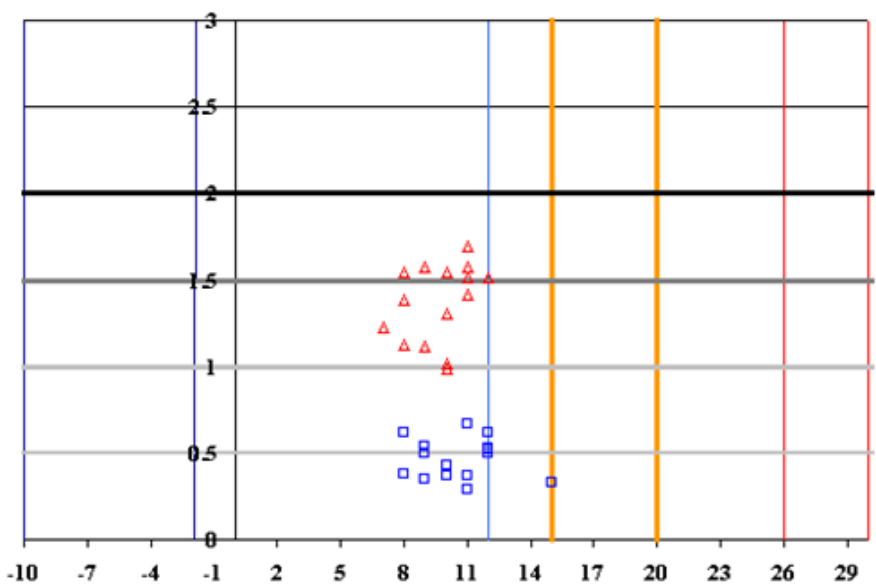

red triangle $=$ Medium daily values collected in Castelnuovo station blue square $=$ Medium daily values collected in Boccadifalco station

Figure 1. Nomo-grams: Thermo-atmospheric assessing 
The previous figure characterizes, for each of the two localities, the environmental exposition and allows to check out the potential effects on the well-being of the exposed population. The colored vertical lines of the nomo-gram delimit the ranges of bio-meteorological feelings. In particular, the lines on the left represent the discomfort from cold while the red ones on the right characterize the discomfort from hot[15].

In the central part, with the two orange lines is delimited the comfort zone (the values range from THI 15 to $20 \mathrm{C}^{\circ}$ ). The horizontal lines, colored from grey to the black, proceeding from the bottom to the top, indicate the ranges of physiological sensation linked to the composite index AQSI. The lower line (grey clear) corresponding to AQSI $=0.5$, represents the limit within which is present the comfort. [16-17]. The black line, corresponding to AQSI=2 indicates the limit of heavy stress. An important characteristic of this representation is the immediacy of the analysis and of the judgment. In both the stations the climatic comfort conditions are never reached but the strong discomfort level is quite negligible.

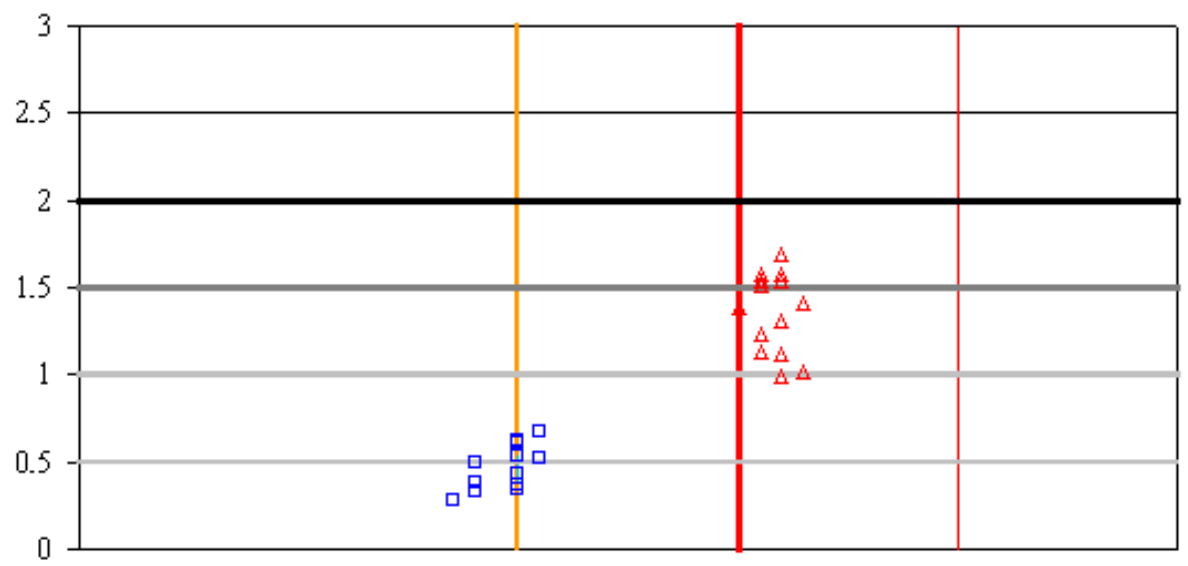

red triangle $=$ Medium daily values collected in Castelnuovo station blu square $=$ Medium daily values collected in Boccadifalco station

Figure 2. Nomograms: Acoustical-atmosferic comfort evaluation

In Fig. 2 it is shown the same approach for the acoustic comfort evaluation. The colored vertical lines delimitate the ranges of acoustical sensation. In particular, shifting from the left to the right is possible to remark the decreasing acoustical comfort. The red vertical line (70 dBA) represents the threshold of acoustical acceptability. The horizontal lines, colored from grey to the black, proceeding from the bottom to the top, indicate the ranges of physiological sensation linked to the composite index AQSI. The lower line (grey clear) corresponding to AQSI=0.5, represents the limit within which is present the comfort. The black line, corresponding to AQSI=2 indicates the limit of heavy stress.

Conclusions. In this paper an operative procedure aimed at assessing and control the global environmental quality along the urban areas is proposed. This methodology is set up using an ergonomic approach that analyzes the interaction man-environment in terms of cause-effect and uses information and data from various aspects contributing to the formation of discomfort degrees. This methodology guarantees to have a very direct and simple evaluation of all parameters involved in human comfort. The mathematical tools used are general and applied in other fields of sciences. The methodology and the representation technique by nomo-grams is experimentally verified on data obtained along the urban territory of Palermo

Acknowledgment. This work was funded by Ministero dell' Ambiente e Del Mare through IMPETUS project. 


\section{References}

[1] Longhetto, A.; Giacomelli, L.; Giraud, C.; Zaramella, G. A study of correlation among solar energy, atmospheric turbidity and pollutants in urban area. Atmospheric Environment 1992, 26B, 29-43.

[2] Barbaro, S.; Costanzo, S.; Grippali, V.; Nicoletti, F. R. A graphical procedure for assessing the global comfort of people in outdoor spaces., Proceedings of the 2nd European Conference REBUILD The European Cities of Tomorrow, Florence, Italy, 1998

[3] Barbaro, S, Bonanno, A.; Chisesi, R, M,; Costanzo, S.; Franzitta, V Grippali, V. Clima e salute nel centro urbano di Palermo, Proceedings $54^{\circ}$ congresso nazionale ATI, L'Aquila, Italy, September 1999.

[4] Barbaro, S, Bonanno, A.; Grippali. . An assessment of light and sound fields for improving the outdoor global comfort., Proceedings of the 3nd European Conference REBUILD The European Cities of Tomorrow, Barcelona, Spain, October 1999.

[5] Franzitta, V., Rizzo, G.. - Renewable energy sources: A mediterranean perspective. ICBEE 2010-2nd International Conference on Chemical, Biological and Environmental Engineering, Proceedings, p.48-51, art. no. 5652332 (2010).

[6] Bonanno, A., Franzitta, V., Muzio, F.P., Trapanese, M. - A multiphysics approach to the design of a seawave energy conversion system. Proceedings of ICSET, Singapore, 2008,p.p. 665 - 668, art. no. 4747090

[7] Di Dio, V., Franzitta, V., Muzio, F., Scaccianoce, G., Trapanese, M. - The use of sea waves for generation of electrical energy and hydrogen. MTS/IEEE Biloxi - Marine Technology for Our Future: Global and Local Challenges, OCEANS, 2009, art. no. 5422319.

[8] Sorrentino, G., Scaccianoce, G., Morale, M., Franzitta, V. The importance of reliable climatic data in the energy evaluation. Energy 2012.

[9] Trapanese, M., Viola, A., Franzitta, V. Description of hysteresis of nickel metal hybride battery. IECON 2012, 38th Annual Conference on IEEE Industrial Electronics Society, Montreal, Canada, pp. 967-970.

[10] Franzitta, V., Viola A., Trapanese M.; Description of hysteresis in Lithium battery by classical Preisach model, Advanced Materials Research Vols. 622-623 (2013) pp 1099-1103.

[11] Ciulla G., Franzitta V.; Lo Brano V., Viola A., Trapanese M., Mini Wind Plant to Power Telecommunication Systems: a Case Study in Sicily, Advanced Materials Research Vols. 622-623 (2013) pp 1078-1083.

[12] Trapanese M., A model of a linear synchronous motor based on distribution theory, J App Phys, Vol.111 (2012), Article number07E731.

[13] Trapanese M, "Noise enhanced stability in magnetic systems", J. App. PhysVolume 105, Issue 7, 2009, Article number 07D313.

[14] Cirrincione M., Miceli R., Galluzzo G.R., M. Trapanese, Preisach function identification by neural networks, IEEE Transactions on Magnetics Volume 38, Issue 5 I, (2002), Pages 2421-2423

[15] A. Messineo, "R744-R717 cascade refrigeration system: performance evaluation compared with a HFC two-stage system", Energy Procedia 14 (2012), 56-65.

[16] Franzitta, V., La Gennusa, M. , Peri, G., Rizzo, G., Scaccianoce, G.. Toward a European Eco-label brand for residential buildings: Holistic or by-components approaches?. Energy Volume 36, Issue 4, (2011), pp 1884-1892.

[17] Trapanese M.; Franzitta V., Viola A. The Jiles Atherton Model for Description Of Hysteresis in Lithium Battery. Conference Proceedings - IEEE Applied Power Electronics Conference and Exposition - APEC 2013- Long Beach,(CA), March 2013-978-1-4673-4355-8, pp 2773-2775. 